\title{
Simultaneous imaging of magnetic field and temperature using a wide-field quantum diamond microscope
}

\author{
Yulei Chen ${ }^{1,2}$, Zhonghao $\mathrm{Li}^{2}$, Hao Guo ${ }^{2}$, Dajin $\mathrm{Wu}^{2}$ and Jun Tang ${ }^{2 *}$
}

${ }^{\text {*Correspondence: }}$

tangjun@nuc.edu.cn

${ }^{2}$ Science and Technology on Electronic Test \& Measurement Laboratory, North University of China, Taiyuan, Shanxi 030051,

China

Full list of author information is available at the end of the article

\begin{abstract}
Quantum sensing based on nitrogen-vacancy centers in diamond has shown excellent properties. Combined with the imaging technique, it shows exciting practicability. Here, we demonstrate the simultaneously imaging technique of magnetic field and temperature using a wide-field quantum diamond microscope. We describe the operating principles of the diamond microscope and report its sensitivity (magnetic field $\sim 1.8 \mu \mathrm{T} / \mathrm{Hz}^{1 / 2}$ and temperature $\sim 0.4 \mathrm{~K} / \mathrm{Hz}^{1 / 2}$ ), spatial resolution $(1.3 \mu \mathrm{m})$, and field of view $\left(400 \times 300 \mu \mathrm{m}^{2}\right)$. Finally, we use the microscope to obtain images of an integrated cell heater and a PCB, demonstrating its ability in the application of magnetic field and temperature simultaneously imaging at wide-field.
\end{abstract}

\section{Introduction}

The techniques such as thermo-reflectance microscopy [1], infrared microscopy (IR) [2], micro-Raman spectroscopy [3] and so on are widely used to measure temperature meanwhile magnetic force microscopy (MFM) [4], superconducting quantum interference devices (SQUIDs) [5], Hall effect sensors [6] and others for the magnetic field measuring. However, the techniques cannot measure the magnetic field and temperature at the same time. At present, it is of great significance to simultaneously image magnetic fields and temperature in many aspects, e.g. material dynamics, carrier transport (nanorobot) and the chip detection [7-12].

Nowadays, solid-state electronic spins in diamond have garnered increasing relevance in sensing. In particular, a negatively charged nitrogen-vacancy (NV) color center in diamond [13] has demonstrated competitive sensing sensitivity in ambient room temperature environments and employed in various applications benefiting from its long spin coherence times at room temperature and the optical initialization and readout of NV spin states by precisely and optically detected magnetic resonance (ODMR) [14]. For magnetic fields and temperature sensing, techniques based on NV color centers have been shown outstanding sensitivity and spatial resolution and developed for biology [15], current wires [16], paramagnetic molecules [17] and so on.

(c) The Author(s) 2021. This article is licensed under a Creative Commons Attribution 4.0 International License, which permits use sharing, adaptation, distribution and reproduction in any medium or format, as long as you give appropriate credit to the original author(s) and the source, provide a link to the Creative Commons licence, and indicate if changes were made. The images or other third party material in this article are included in the article's Creative Commons licence, unless indicated otherwise in a credit line to the material. If material is not included in the article's Creative Commons licence and your intended use is not permitted by statutory regulation or exceeds the permitted use, you will need to obtain permission directly from the copyright holder. To view a copy of this licence, visit http://creativecommons.org/licenses/by/4.0/. 
In addition, measuring not only the value of the field intensity but also the full intensity image of the sample is particularly valuable. The technique combined ODMR and imaging can not only support a combination of superior spatial resolution, sensing sensitivity, and wide field of view but also the ability to produce a full-field image of the sample [13]. Also, the imaging technique using spins in diamond does not need prolonged data acquisition as MFM and can be performed at room temperature compared with electron and $\mathrm{x}$-ray microscopy, SQUID, and so on [18]. These advantages make it a powerful imaging tool for magnetic field and temperature detection especially in the situation that wide-field, high-spatial resolution and real-time are needed.

The NV center sensing exhibits excellent performance in magnetic fields and temperature and combining with the imaging technique is a very promising tool in application. The work presented here therefore investigates simultaneous imaging of magnetic field and temperature for the chip detection using NV color centers in diamond. Firstly, it is carried out by describing the properties of NV color centers and the ODMR spectrum. And then the principle of magnetic fields and temperature imaging with NVs is explained. Finally, the experimental results of magnetic fields and temperature of an integrated cell heater [19] widely used in temperature control with magnetic fields suppression are shown, followed by magnetic fields imaging of a Printed Circuit Board (PCB) wire and temperature imaging of solder joints. The experimental results indicated that the NV imaging technique can be effectively used for simultaneously image magnetic fields and temperature, and therefore can be also used to chip failure detection and so on.

\section{Principle and experimental setup}

The experimental setup is illustrated in Fig. 1(a). The diamond is illuminated with a $532 \mathrm{~nm}$ laser beam through the self-assembled confocal wide-field imaging system [20] and the resulting fluorescence was collected via the objective lens and filtered to pass light of 650-800 nm before being imaged by a scientific complementary metal oxide semiconductor (sCMOS) camera. The laser power density used for our imaging system is $10 \mathrm{~W} / \mathrm{mm}^{2}$. A resonance microwave field with a power of $20 \mathrm{dBm}$ is fed to diamond via microwave antenna. External magnetic field $\left(B_{0}=7.5 \mathrm{mT}\right)$ is used to provide the NV centers with a known magnetic bias. The device under test (DUT) is placed under the diamond. The CVD diamond chip used in our experiment was a (100) oriented plate such that the normal vector to the bottom surface (sensing plane) was parallel to the [100] crystallographic direction. The diamond is polished to be $50 \mu \mathrm{m}$ thick and estimated the concentrations of NV center to be about $1 \mathrm{ppm}$ [21].

As shown in Fig. 1(b), NV color center is an atomic-scale defect which consists of a substitutional nitrogen atom adjacent to a vacancy in the diamond lattice. And the operating principle of the NV-based imaging technique relies on the spin-triplet ground state $\left({ }^{3} \mathrm{~A}_{2}\right)$ of the NV structure which is split in energy by spin-spin interaction into a singlet state $m_{S}=0$ and a doublet state $m_{S}= \pm 1$, separated by $D=2.87 \mathrm{GHz}$ in the absence of magnetic field (zero-field splitting) [22]. In the presence of an external magnetic field, the $m_{S}= \pm 1$ sublevels are shifted due to the Zeeman effect. From the Hamiltonian of the NV-centers, applying a bias magnetic field that the frequencies $f_{ \pm}$of $m_{S}=0 \rightarrow m_{S}= \pm 1$ transitions can be given by [22]:

$$
f_{ \pm}=D+\beta_{T} \delta_{T} \pm \gamma B_{\mathrm{NV}}
$$




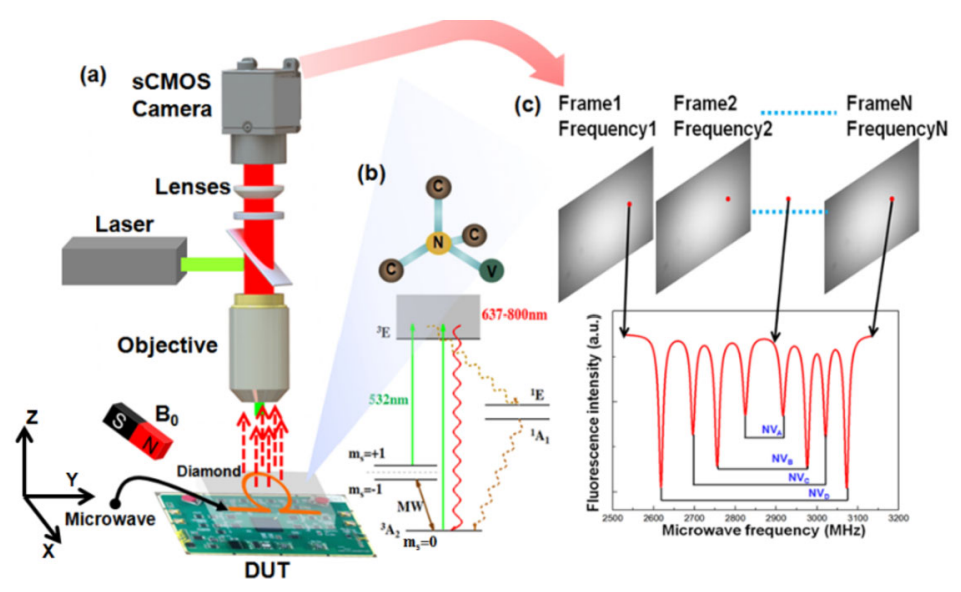

Figure 1 (a) The wide-field quantum diamond microscope used for magnetic fields and temperature imaging. (b) Atomic structure and energy-level diagram of the NV defect. (c) Schematic diagram of the ODMR scan imaging method. The camera captures $\mathrm{N}$ frames pictures synchronizing with microwave steps. Magnetic fields and temperatures were carried out by using the frequency shift of NVa, b, b, and $d$

where $f_{ \pm}$are the NV resonance frequencies corresponding to the $m_{S}=0 \rightarrow m_{S}= \pm 1$ transitions, $\beta_{T} \approx-74 \mathrm{kHz} / \mathrm{K}$ at room temperature [23], $\delta_{T}$ is the temperature change relative to $300 \mathrm{~K}$ during the measurement, $\gamma=g_{e} \mu_{B} / h \approx 28 \mathrm{~Hz} / \mathrm{nT}$ is the $\mathrm{NV}$ gyromagnetic ratio [22], and $B_{N V}$ is the projection of the applied magnetic field along the NV symmetry axis. The NV center will be polarized with optically pumped exhibiting a high fluorescence rate. When specific microwave energy is applied,the center is excited in the $m_{S}= \pm 1$ levels and the NVs will exhibit a higher probability to transfer to the non-radiative singlet state followed by a decay to $m_{S}=0$. As a result, the spin state can be detected by optically detected magnetic resonance (ODMR).

The diamond is imaged on the focal plane of the sCMOS camera with a pixel size of $\sim 5 \mu \mathrm{m}$. The camera is synchronized to the microwave source which is swept from 2.5 to $3.2 \mathrm{GHz}$ in 4000 steps. And then, we acquire a stack of images and each image represents a microwave frequency. The method of image acquisition is shown in Fig. 1(c). For each pixel, all pictures constitute the completed ODMR spectrum. The spectra comprise eight lines due to the spin resonances for each of the four possible NV orientations (NVa to d). These eight lines are resolved via the application of an oriented bias magnetic field $B_{0}$ [24]. By simultaneously measuring the frequencies shifts of the resonance frequencies of all four possible $\mathrm{NV}$ axes, the effects of temperature and magnetic field can be decoupled and thus one can achieve magnetic field $\left(B_{\mathrm{a} . . . \mathrm{d}}\right)$ and temperature $\left(\delta_{\mathrm{a} \ldots \mathrm{d}}\right)$ measurements as shown in equation (1).

We define the laboratory reference frame with $\mathrm{x}$ and $\mathrm{y}$ in the plane of the diamond surface and $\mathrm{z}$ perpendicular to the plane as shown in Fig. 1(a). In the coordinates, unit vectors for the [111] crystal directions of the four NV orientations can be defined as follows:

$$
\begin{array}{ll}
\vec{u}_{a}=\left(-\sqrt{\frac{2}{3}}, 0, \sqrt{\frac{1}{3}}\right), & \overrightarrow{u_{b}}=\left(\sqrt{\frac{2}{3}}, 0, \sqrt{\frac{1}{3}}\right), \\
\vec{u}_{c}=\left(0, \sqrt{\frac{2}{3}}, \sqrt{\frac{1}{3}}\right), & \overrightarrow{u_{d}}=\left(0,-\sqrt{\frac{2}{3}}, \sqrt{\frac{1}{3}}\right) .
\end{array}
$$


For magnetic fields $B_{\mathrm{a}}$ to $B_{\mathrm{d}}$, corresponding to frequency shift measurements $\left(\left(f_{+}-f_{-}\right) / 2\right)$ along axes a to $\mathrm{d}$, respectively, the fields in the laboratory Cartesian coordinates can be calculated from:

$$
\left\{\begin{array}{l}
B_{x}=\frac{\sqrt{6}}{4}\left(B_{\mathrm{b}}-B_{\mathrm{a}}\right), \\
B_{y}=\frac{\sqrt{6}}{4}\left(B_{\mathrm{c}}-B_{\mathrm{d}}\right), \\
B_{z}=\frac{\sqrt{3}}{4}\left(B_{\mathrm{a}}+B_{\mathrm{b}}+B_{\mathrm{c}}+B_{\mathrm{d}}\right) .
\end{array}\right.
$$

For temperature $\delta_{\mathrm{a}}$ to $\delta_{\mathrm{d}}$, corresponding to frequency measurements $\left(\left(f_{+}+f_{-}-2 D\right) / 2\right)$ along axes a to $\mathrm{d}$, respectively, temperature can be calculated from:

$$
T=\frac{1}{4}\left(\delta_{\mathrm{a}}+\delta_{\mathrm{b}}+\delta_{\mathrm{c}}+\delta_{\mathrm{d}}\right) .
$$

\section{Results and discussion}

Integrated cell heaters are widely used in a variety of sensors operating at high temperatures such as gas sensors [25], micro nuclear magnetic resonance (NMR) gyroscope [19] and so on. For sensors based on NMR, it is of great significance to understand the distribution of the magnetic field and temperature of the cell heaters. Therefore we used the imaging system to detect the magnetic field and temperature produced by integrated cell heater in ref. [19]. Figure 2(a) is a sketch map of the integrated cell heater. The inset shows the optical photograph of the part of the heater to be tested. The heater is designed to temperature control with magnetic fields suppression utilized current carrying with (+--+-++-) configuration.

In the experiment, to obtain a larger field of view (FOV), a $10 \times / 0.3$ microscope objective was applied at first. Based on the size of the laser spot in our system, we got $800 \times 600$ pixels FOV on the camera corresponding to $400 \times 300 \mu \mathrm{m}^{2}$ imaging area. And the spatial resolution is to be $1.3 \mu \mathrm{m}$ mainly restricted by the optical diffraction limit. The exposure time for each fluorescence image was $8 \mathrm{~ms}$, with a total integration time of $42 \mathrm{~ms}$ per microwave frequency, corresponding to a total measurement time 200 seconds. Another important characteristic of the imaging technique is its sensitivity. The magnetic field optimal photon shot noise-limited sensitivity can be expressed as [22]:

$$
\eta \approx \frac{h}{g \mu_{B}} \frac{\Delta V}{C \sqrt{I_{0}}}
$$

where $h$ is the Planck constant, $g$ the Lande factor, $\mu_{B}$ the Bohr magneton, $I_{0}=3.4 \times 10^{8} \mathrm{~s}^{-1}$ the number of photons per detection volume, $\Delta V=12 \mathrm{MHz}$ the ODMR linewidth and $C=2.8 \%$ the ODMR contrast giving a magnetic sensitivity of $1.8 \mu \mathrm{T} / \mathrm{Hz}^{1 / 2}$.

Based on the parameters mentioned above, Fig. 2(a) and Fig. 2(b) show the simulation and detection results of the magnetic field with changing currents on the heater surface, respectively. When the heater is not working (current $=0$ ), the fluctuations of the magnetic field imaging is due to fitting errors and system noises. For the fluctuation, we subtracted it from the test results to reduce or even eliminate the errors. With the increase of current, the change of magnetic field intensity is clearly characterized by the imaging system and we note strong agreement between measurements and simulations and we attribute the small deviations from the simulations to the inadequate precision of the heater processing. 
(a)

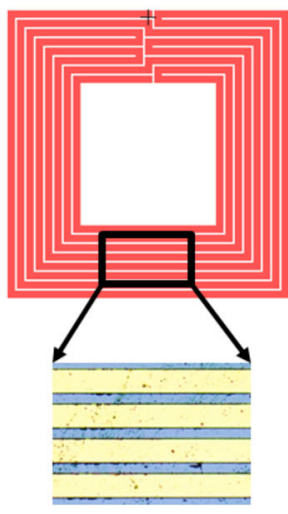

(b)

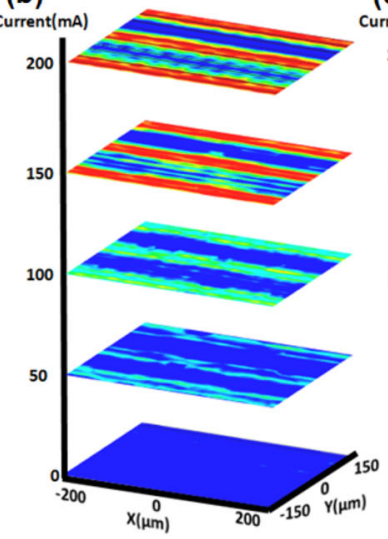

(c)

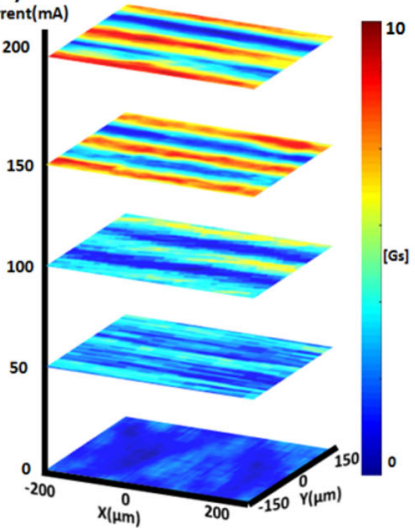

Figure 2 (a) The sketch map of the integrated cell heater. The inset shows the optical photograph of the part of the heater to be tested. (b) and (c) The simulation and test results of magnetic field distribution with changing currents in the imaging region, respectively

(a)

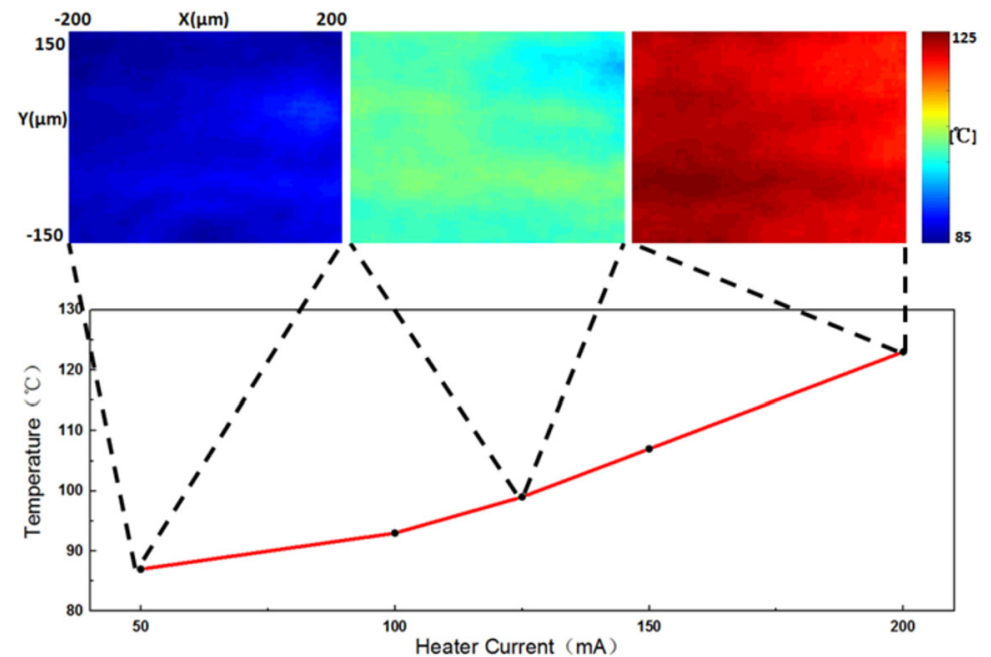

(b)

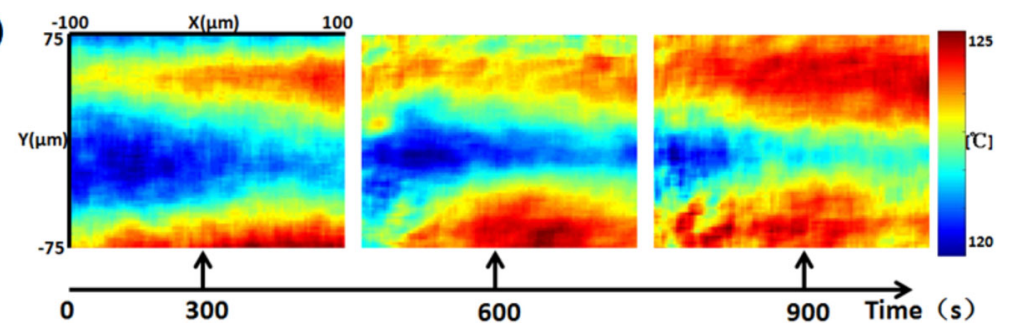

Figure 3 (a) The bottom: the temperature as a function of the current measured by a thermometer. The top: three temperature images corresponding to different currents under the same scale. (b) The thermal diffusion imaging of two wires under the current of $200 \mathrm{~mA}$

For the temperature detection, the sensitivity is also fundamentally limited by $I_{0}, \Delta V$ and $C$ [26], thus giving a temperature sensitivity of $0.4 \mathrm{~K} / \mathrm{Hz}^{1 / 2}$.

The bottom of Fig. 3(a) shows the temperature as a function of the current measured by a thermometer. Using the $10 \times / 0.3$ microscope objective, temperature imaging is im- 
(a)

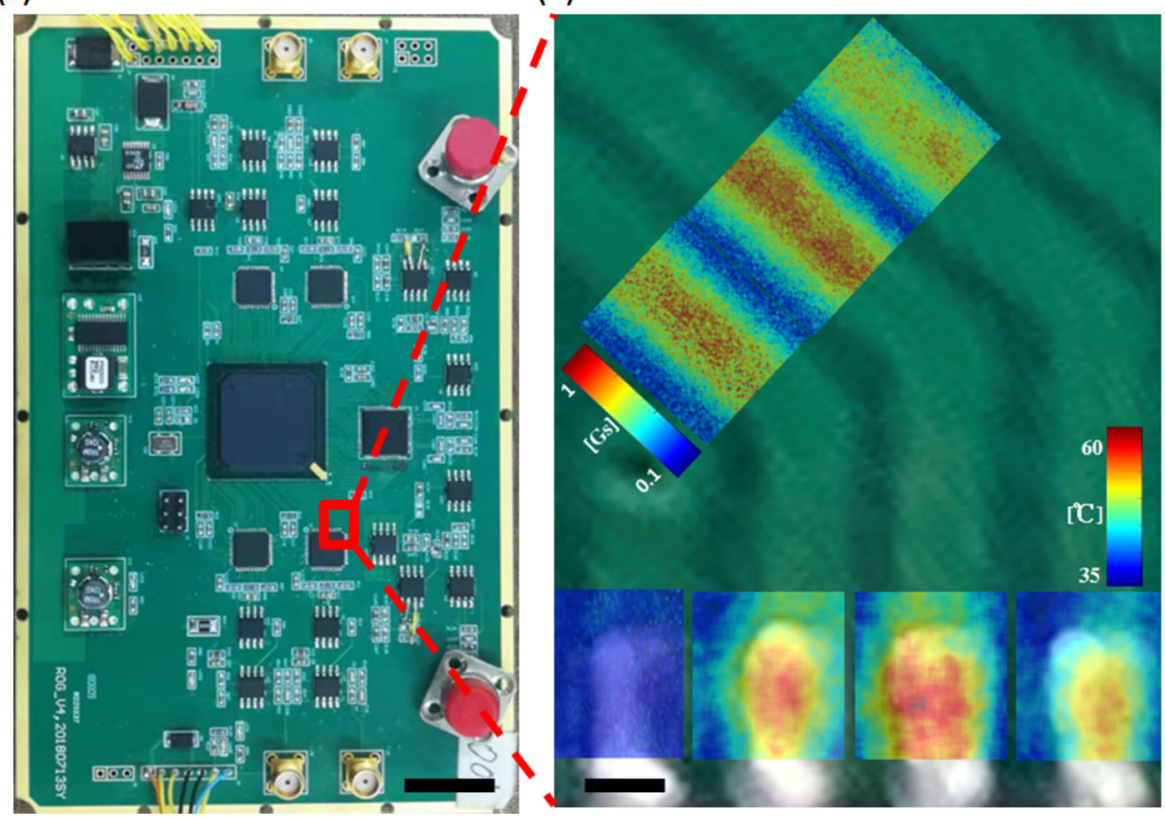

Figure 4 (a) The PCB prototype photograph. (b) Magnetic fields imaging of the wires and temperature imaging of the solder joints. Scalebars, (a) $2 \mathrm{~cm}$ and (b) $20 \mu \mathrm{m}$

plemented. Three temperature images under the same scale corresponding to different currents are displayed at the top of Fig. 3(a). The pictures show obvious temperature difference, but the details cannot be distinguished due to the large temperature span. In order to better distinguish and observe the temperature change, we used $20 \times / 0.4$ objective and observed the phenomenon of the thermal diffusion of two wires under the current of $200 \mathrm{~mA}$. Figure 3(b) shows the test results and the phenomenon of thermal diffusion can be seen clearly. The experimental results indicate that NVs-based imaging technology has the ability of real-time chip-working monitoring.

Further, we have carried out experiments on a PCB used in our laboratory. Figure 4(a) shows the PCB prototype photograph. The area marked in red was tested. Imagings of wire magnetic fields and solder joints temperature are shown in Fig. 4(b), respectively. From the magnitude of the magnetic field, we can infer whether the wire is conductive or not and even the magnitude of the current. It can be judged from the imaging result that the current passed through the middle conductor is the maximum. The temperature of the corresponding solder joint is also the highest. The current density can be estimated by the temperature distribution. And also it can be used to judge whether the solder joint is faulty like the leftmost solder joint. These results elucidate that the quantum diamond NVsbased microscope has a bright future in the application of magnetic field and temperature simultaneously imaging at wide-field such as quality control of PCB.

\section{Conclusion}

In this letter, a wide-field NVs-based microscope for simultaneous imaging of magnetic field and temperature has been proposed, experimentally studied and discussed. The experimental results show that the imaging technique based on NV color centers is a powerful tool for simultaneous imaging the magnetic field and temperature distribution es- 
pecially in the situation that wide-field, high-spatial resolution and real-time are needed. Moreover, a number of technical improvements which could enhance the performance of the microscope such as using dynamical decoupling pulse sequences, lock-in camera and so on make it a more potential technique to help us to detected weaker signals such as failure mechanisms of devices and bio-information.

\section{Acknowledgements}

The authors are grateful for the use of the equipment of the Science and Technology on Electronic Test \& Measurement Laboratory.

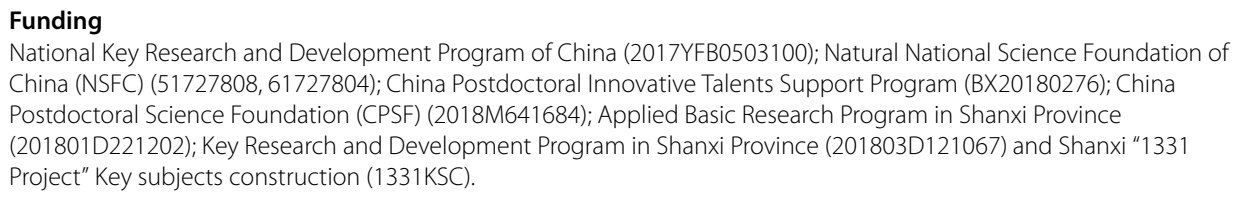

Availability of data and materials

The data that support the findings of this study are available from the corresponding author upon reasonable request.

\section{Competing interests}

The authors declare that they have no competing interests.

\section{Authors' contributions}

Conceptualization, YLC and JT; software, ZHL; validation, HG; formal analysis, DJW; investigation, YLC; data curation, YLC; writing —original draft preparation, YLC; writing — review and editing, ZHL; funding acquisition, JT. All authors read and approved the final manuscript.

\section{Author details}

${ }^{1}$ College of Physics and Information Engineering, Shanxi Normal University, Linfen, Shanxi 04100, China. ${ }^{2}$ Science and Technology on Electronic Test \& Measurement Laboratory, North University of China, Taiyuan, Shanxi 030051, China.

\section{Publisher's Note}

Springer Nature remains neutral with regard to jurisdictional claims in published maps and institutional affiliations.

Received: 13 November 2020 Accepted: 17 March 2021 Published online: 25 March 2021

\section{References}

1. Heiderhoff R, Makris A, Riedl T. Mater Sci Semicond Process. 2016;43:163-76.

2. Dazzi A, Prater CB. Chem Rev. 2016;117:5146-73.

3. Park KD, Muller EA, Kravtsov V, Sass PM, Dreyer J, Atkin JM, Raschke MB. Nano Lett. 2015:16:479-87.

4. Shinjo T, Okuno T, Hassdorf R, Shigeto K, Ono T. Science. 2000;289:930-2.

5. Ulusar U, Wilson J, Murphy P, Govindan R, Preissl H, Lowery C, Eswaran H. Physiol Meas. 2011;32:263.

6. Damsgaard CD, Dalslet BT, Freitas SC, Freitas PP, Hansen MF. Sens Actuators A, Phys. 2009;156:103-8.

7. Colliex C. Science. 2015;347:611-2.

8. Hu Y, Zeng L, Minnich AJ, Dresselhaus MS, Chen G. Nat Nanotechnol. 2015;10:701.

9. Shi L, Plyasunov S, Bachtold A, McEuen PL, Majumdar A. Appl Phys Lett. 2000;77:4295-7.

10. Hassan MT, Baskin J, Liao B, Zewail A. Nat Photonics. 2017;11:425.

11. Pop E, Sinha S, Goodson KE. Proc IEEE. 2006;94:1587-601.

12. Bagnall KR, Moore EA, Badescu SC, Zhang L, Wang EN. Rev Sci Instrum. 2017:88:113111.

13. Doherty MW, Manson NB, Delaney P, Jelezko F, Wrachtrupe J, Hollenberg LCL. Phys Rep. 2013;528:1.

14. El-Ella HA, Ahmadi S, Wojciechowski AM, Huck A, Andersen UL. Opt Express. 2017;25:14809-21.

15. Fedotov I, Safronov N, Ermakova YG, Matlashov M, Sidorov-Biryukov D, Fedotov A, Belousov V, Zheltikov A. Sci Rep. 2015;5:15737.

16. Chen EH, Clevenson HA, Johnson KA, Pham LM, Englund DR, Hemmer PR, Braje DA. Phys Rev A. 2017;95:053417.

17. Mamin H, Kim M, Sherwood M, Rettner C, Ohno K, Awschalom D, Rugar D. Science. 2013;339:557-60.

18. Davis HC, Ramesh P, Bhatnagar A, Lee-Gosselin A, Barry JF, Glenn DR, Walsworth RL, Shapiro MG. Nat Commun. 2018;9:131.

19. Noor RM, Shkel AM. J Microelectromech Syst. 2018:27:1148-59.

20. Chen Y, Guo H, Li W, Wu D, Zhu Q, Zhao B, Wang L, Zhang Y, Zhao R, Liu W. Appl Phys Express. 2018;11:123001.

21. Zhu Q, Guo H, Chen Y, Wu D, Zhao B, Wang L, Zhang Y, Zhao R, Du F, Tang J. Jpn J Appl Phys. 2018;57:110309.

22. Rondin L, Tetienne J-P, Hingant T, Roch J-F, Maletinsky P, Jacques V. Rep Prog Phys. 2014;77:056503.

23. Acosta VM, Bauch E, Ledbetter MP, Waxman A, Bouchard L-S, Budker D. Phys Rev Lett. 2010;104:070801.

24. Tetienne JP, Dontschuk N, Broadway D, Lillie S, Teraji T, Simpson D, Stacey A, Hollenberg L. Phys Rev B. 2019;99:014436.

25. Behera B, Chandra S. Sens Actuators B, Chem. 2016;229:414-24.

26. Schirhagl R, Chang K, Loretz M, Degen CL. Annu Rev Phys Chem. 2014;65:83-105. 